\title{
Divergence of the IGS rDNA in Fusarium proliferatum and Fusarium globosum reveals two strain specific non-orthologous types
}

\author{
Miguel Jurado • Patricia Marín • Covadonga Vázquez • \\ M. Teresa González-Jaén
}

\begin{abstract}
A phylogenic analysis of Fusarium proliferatum and closely related species was performed using the most variable part within the intergenic spacer of the nuclear ribosomal DNA (IGS) and compared with a previously reported phylogeny performed in the same group of samples with a partial region of the nuclear single copy gene encoding the elongation factor $1 \alpha(E F-l \alpha)$. The phylogenies from both genomic sequences were not concordant and revealed the presence of two nonorthologous IGS types, named types I and II, in $F$. proliferatum and Fusarium globosum.

Two specific PCR assays designed to amplify either IGS type I or type II revealed that only one IGS type was present in each individual in these two species. The presence of both IGS types at the species level indicates that homogenization has not been achieved yet. This might be retarded if panmictic sexual reproduction was affected by certain levels of clonal reproduction and/or by the diverse hosts that these species are able to colonize. This study indicates that taxonomic studies carried out with the
\end{abstract}

IGS rDNA, which has been widely used in Fusarium, should be undertaken with caution.

Keywords Fusarium proliferatum . Gibberella fujikuroi . Genetic variability $\cdot$ Non-orthologous $\cdot$ IGS $\cdot$ EF-1 $\alpha$

\section{Introduction}

Fusarium proliferatum (Gibberella intermedia) is an important pathogen which colonizes a wide range of agricultural plant species, and it is capable of producing fumonisins, among other secondary metabolites, which can accumulate in food and feed products causing serious chronic and acute diseases in human and animals. This species is a member of the Gibberella fujikuroi species complex, the latter of which was resolved in several distinct anamorphs by phylogenetic analyses using genomic DNA sequences (O'Donnell et al. 1998). Distinct anamorphs were also proposed for some of these species (Nirenberg and O'Donnell 1998) and currently, the Gibberella fujikuroi species complex is considered as a monophyletic taxon, including nine sexually fertile biological species or teleomorphs, also known as mating populations (MP-A to MP-I), and more than 30 anamorphs in the genus Fusarium (Nirenberg and O'Donnell 1998).

In the last two decades, DNA-based methods have helped to elucidate relationships among different species of Fusarium, which had been previously explored based exclusively on morphological features and/or mating experiments. The introduction of the phylogenetic species concept has proven to be very useful in fungal systematics, particularly to members of the G. fujikuroi species complex, resolving distinct species with characteristic toxigenic profiles and providing the basis to develop diagnostics 
based on phylogenetic species. This concept relies in a major gene-gene concordance which allows inferring strict consensus phylogenetic trees from the combined data sets (Geiser et al. 2004), whereas the lack of concordance among different DNA data sets might reflect incomplete genetic isolation. Among the DNA sequences more frequently analysed for taxonomic purposes, the nuclear ribosomal DNA (rDNA) sequences have been extensively employed to infer phylogenies in a wide number of taxa, including the genus Fusarium. The rDNA genes are organized in units located in tandem (Smith 1976) and separated by non-coding spacer regions of variable length. In filamentous fungi, the rDNA genes encoding $18 \mathrm{~S}, 5.8 \mathrm{~S}$ and $28 \mathrm{~S}$ genes, are highly conserved whereas the internal transcribed spacers (ITS) located between the $18 \mathrm{~S}$ and $5.8 \mathrm{~S}$ genes (ITS1) and between the $5.8 \mathrm{~S}$ and $28 \mathrm{~S}$ (ITS2) genes, and the intergenic spacer (IGS) residing between the $28 \mathrm{~S}$ and $18 \mathrm{~S}$ genes, exhibit a high inter and intraspecific variability. This makes them useful for phylogenetic studies. The individual repeats, including ITS and IGS spacers and the rDNA unit, evolve in concert rather than independently and it is believed to result from a phenomenon (concerted evolution) that homogenizes all the repeats in the array and fix the rDNA repeat units within a sexually reproducing species (Dover 1982). The major mechanisms considered to account for concerted evolution are unequal crossing-over (mainly between sister chromatids) and gene conversion, although evidences for the latter are still scarce (Eickbush and Eickbush 2007). Concerted evolution permits phylogeny reconstruction based on rDNA sequences and only few exceptional cases might have escaped concerted evolution, such as the non-concerted pattern of ITS2 sequences in the G. fujikuroi species complex (Waalwijk et al. 1996; O'Donnell and Cigelnik 1997; O'Donnell et al. 1998), making this region ineffective for phylogenetic inference of these species (Kvas et al. 2009). This, however, has not been described so far for the IGS region which has been broadly used for taxonomic purposes in the genus Fusarium (Kosiak et al. 2005; Tóth et al. 2005) and, particularly, in the species of the G. fujikuroi complex (Mirete et al. 2004; Yli-Mattila et al. 2004; Alves-Santos et al. 2007). The species included in this complex are considered reproductively isolated (O'Donnell et al. 1998), although in the case of $F$. proliferatum and the most closely related species, the reproductive barrier might not has been completely formed (Xu et al. 1995).

In this work, we studied the phylogenetics of $F$. proliferatum and its most closely related species using a partial sequence corresponding to the most variable part within the IGS region. This phylogeny was compared with that previously reported in the same group of samples with a partial region of the nuclear single copy gene encoding the elongation factor $1 \alpha(E F-1 \alpha)$ (Jurado et al. 2010). In particular, we examined the concordance of topologies between both types of sequences and presence of nonorthologous loci for the IGS rDNA.

\section{Material and methods}

Fungal isolates

The fungal strains used in this study belonged to the following closely related species: $F$. proliferatum, Fusarium globosum, Fusarium concentricum, Fusarium fractiflexum and Fusarium fujikuroi (Table 1). An IGS rDNA sequence of Fusarium verticillioides was included as an outgroup for the phylogenetic studies described below. Cultures were maintained on potato dextrose agar medium (PDA) (Scharlau Chemie, Barcelona, Spain) at $4^{\circ} \mathrm{C}$ and stored as spore suspensions in $15 \%$ glycerol at $-80^{\circ} \mathrm{C}$.

\section{DNA isolation, PCR amplification and sequencing}

Genomic DNA was extracted from fungal cultures with a Genomix DNA extraction kit (Talent, Italy) according to the manufacturer's instructions.

PCR amplification of a portion of thel IGS region was carried out with primers Gib2-F (5' GAGGCGCGGT GTCGGTGTGCTTG $3^{\prime}$ ) and Fgc-R (5' CTCTCATA TACCCTCCG 3') (Jurado et al. 2006). The amplification protocol was 1 cycle of $85 \mathrm{~s}$ at $94^{\circ} \mathrm{C}, 25$ cycles of $35 \mathrm{~s}$ at $95^{\circ} \mathrm{C}$ (denaturation), $30 \mathrm{~s}$ at $58^{\circ} \mathrm{C}$ (annealing), $30 \mathrm{~s}$ at $72^{\circ} \mathrm{C}$ (extension), and 1 cycle of $10 \mathrm{~min}$ at $72^{\circ} \mathrm{C}$. This PCR assay amplifies an internal fragment within the IGS region of $\sim 1000 \mathrm{bp}$ (approximately from nucleotides 780 to 1780 ) in all of the species of the Gibberella fujikuroi complex tested.

In all cases, amplification reactions were carried out in volumes of $25 \mu \mathrm{L}$ containing $200 \mathrm{ng}$ of template DNA in $3 \mu \mathrm{L}, 1.25 \mu \mathrm{L}$ of each primer $(20 \mu \mathrm{M}), 0.2 \mu \mathrm{L}$ of Taq DNA polymerase $(5 \mathrm{U} / \mu \mathrm{L}), 2.5 \mu \mathrm{L}$ of $10 \mathrm{X}$ PCR buffer, $1 \mu \mathrm{L}$ of $\mathrm{MgCl} 2(50 \mathrm{mM})$, and $0.25 \mu \mathrm{L}$ of dNTPs $(100 \mathrm{mM})$ (Ecogen, Barcelona, Spain) in a thermocycler (Eppendorf Mastercycler Gradient, Eppendorf, Hamburg, Germany). Amplification products were detected by electrophoresis on $1.5 \%$ agarose ethidium bromide gels in $40 \mathrm{mM}$ Tris-acetate and $1.0 \mathrm{mM}$ EDTA $1 \mathrm{X}$ buffer.

Amplification products of the IGS region were isolated using the High Pure PCR Product Purification Kit (Roche, Mannheim, Germany). Sequencing was performed in an ABI 3700 DNA Sequencer in the Genomic Unit of the Universidad Complutense of Madrid (Madrid, Spain). Sequences were edited and aligned using the Clustal method by Dnastar (Lasergene, Wisconsin, USA), and deposited in the NCBI Genbank database (numbers of accession are shown in Table 1). 
Table 1 Isolates analyzed indicating species, host, origin, MAT allele, occurrence of PCR amplification product using PCR assays type I and type II, and number of accession of partial IGS region sequences deposited in the NCBI Genbank

\begin{tabular}{|c|c|c|c|c|c|c|}
\hline Isolate $^{\mathrm{a}}$ & $\operatorname{Host}^{\mathrm{b}}$ & Origin & $\mathrm{MAT}^{\mathrm{c}}$ & Type I & Type II & Accession number (IGS) \\
\hline \multicolumn{7}{|c|}{ F. proliferatum } \\
\hline Fp101 & Ta & France & 1 & - & + & GQ495206 \\
\hline Fp102 & Ta & France & 1 & - & + & GQ495207 \\
\hline Fp103 & $\mathrm{Ta}$ & France & 1 & - & + & GQ495208 \\
\hline Fp175 & $\mathrm{Ta}$ & France & 1 & + & - & GQ495209 \\
\hline $\mathrm{FpB} 12$ & $\mathrm{Hv}$ & Spain & 2 & + & - & GQ495212 \\
\hline $\mathrm{FpB} 23$ & $\mathrm{Hv}$ & Spain & 2 & + & - & GQ495217 \\
\hline $\mathrm{FpB} 22$ & $\mathrm{Hv}$ & Spain & 1 & - & + & GQ495216 \\
\hline $\mathrm{FpB} 20$ & $\mathrm{Hv}$ & Spain & 1 & - & + & GQ495214 \\
\hline $\mathrm{FpB} 21$ & $\mathrm{Hv}$ & Spain & 1 & - & + & GQ495215 \\
\hline $\mathrm{FpB} 15$ & $\mathrm{Hv}$ & Spain & 2 & + & - & GQ495213 \\
\hline FpO24 & $\mathrm{Zm}$ & Spain & 2 & + & - & GQ495220 \\
\hline $\mathrm{FpC} 24$ & $\mathrm{Zm}$ & Spain & 2 & + & - & GQ495219 \\
\hline $\mathrm{FpC} 3$ & $\mathrm{Zm}$ & Spain & 1 & + & - & GQ495218 \\
\hline FpMM6-2 & $\mathrm{Zm}$ & Spain & 1 & + & - & GQ495199 \\
\hline FpMM4-2 & $\mathrm{Zm}$ & Spain & 1 & + & - & GQ495197 \\
\hline FpMM6-1 & $\mathrm{Zm}$ & Spain & 1 & - & + & GQ495198 \\
\hline FpMM3-1 & $\mathrm{Zm}$ & Spain & 1 & + & - & GQ495195 \\
\hline FpMM1-1 & $\mathrm{Zm}$ & Spain & - & + & - & GQ495193 \\
\hline FpMM1-2 & $\mathrm{Zm}$ & Spain & 1 & + & - & GQ495211 \\
\hline FpMM1-3 & $\mathrm{Zm}$ & Spain & 2 & + & - & GQ495194 \\
\hline FpMM4-1 & $\mathrm{Zm}$ & Spain & 2 & + & - & GQ495196 \\
\hline MPD 4853 & & & 1 & - & + & GQ495221 \\
\hline Gf37 & Ms & Ecuador & 1 & + & - & GQ495205 \\
\hline Gf33 & $\mathrm{P}$ & Spain & 2 & - & + & GQ495203 \\
\hline Gf31 & $\mathrm{P}$ & Spain & 2 & - & + & GQ495202 \\
\hline Gf29 & $\mathrm{P}$ & Spain & 2 & - & + & GQ495201 \\
\hline Gf34 & $P$ & Spain & - & + & - & GQ495204 \\
\hline Gf26 & $\mathrm{P}$ & Spain & 1 & + & - & GQ495200 \\
\hline Fp2287 & & Italy & 2 & + & - & GQ495210 \\
\hline ITEM 1682 & $\mathrm{Zm}$ & Canada & - & + & - & GQ495182 \\
\hline ITEM 4293 & $\mathrm{Ch}$ & Spain & - & + & - & GQ495191 \\
\hline ITEM 2298 & $\mathrm{Zm}$ & Italy & 1 & + & - & GQ495184 \\
\hline ITEM 2191 & $\mathrm{Zm}$ & Italy & 1 & - & + & GQ495183 \\
\hline ITEM 2620 & $\mathrm{Zm}$ & Slovakia & - & + & - & GQ495187 \\
\hline ITEM 1506 & $\mathrm{Zm}$ & Italy & 1 & - & + & GQ495181 \\
\hline ITEM 2644 & $\mathrm{Zm}$ & Slovakia & 2 & + & - & GQ495188 \\
\hline ITEM 4306 & W & Spain & 2 & + & - & GQ495192 \\
\hline ITEM 1456 & Ao & Italy & 2 & + & - & GQ495179 \\
\hline ITEM 1486 & Ao & Italy & - & + & - & GQ495180 \\
\hline ITEM 1451 & Ao & Italy & 1 & + & - & GQ495178 \\
\hline ITEM 4285 & $\mathrm{Pc}$ & Spain & 2 & + & - & GQ495189 \\
\hline ITEM 2343 & $\mathrm{Pd}$ & Saudi Arabia & - & + & - & GQ495186 \\
\hline ITEM 2341 & $\mathrm{Pd}$ & Saudi Arabia & - & + & - & GQ495185 \\
\hline ITEM 4291 & $\operatorname{Pr}$ & Spain & - & + & - & GQ495190 \\
\hline \multicolumn{7}{|l|}{ F. globosum } \\
\hline ITEM 1590 & $\mathrm{Ad}$ & Italy & & - & + & GQ495174 \\
\hline ITEM 1874 & Ad & Italy & & - & + & GQ495175 \\
\hline
\end{tabular}


Table 1 (continued)

\begin{tabular}{|c|c|c|c|c|c|c|}
\hline Isolate $^{\mathrm{a}}$ & Host $^{b}$ & Origin & $\mathrm{MAT}^{\mathrm{C}}$ & Type I & Type II & Accession number (IGS) \\
\hline ITEM 6013 & & & & + & - & GQ495176 \\
\hline NRRL 26131 & & & & + & - & GQ495177 \\
\hline \multicolumn{7}{|l|}{ F. concentricum } \\
\hline NRRL25303 & & & & N.T. & N.T. & GQ495170 \\
\hline \multicolumn{7}{|l|}{ F. fractiflexum } \\
\hline NRRL28852 & & & & N.T. & N.T. & GQ495171 \\
\hline \multicolumn{7}{|l|}{ F. fujikuroi } \\
\hline NRRL13566 & & & & N.T. & N.T. & GQ495172 \\
\hline \multicolumn{7}{|l|}{ F. verticillioides } \\
\hline FvMM2-4 & $\mathrm{Zm}$ & Spain & & N.T. & N.T. & GQ495173 \\
\hline
\end{tabular}

${ }^{a}$ ITEM: strains deposited in microbial collection of Institute of Sciences of Food Production (Bari, Italy); NRRL: strains deposited in microbial collection of Agricultural Research Culture Collection (Peoria, USA); the rest of strains belong to a private collection from the Faculty of Biology, Complutense University of Madrid (Spain) ${ }^{\mathrm{b}}$ Ta: Triticum aestivum; Hv: Hordeum vulgare; Zm: Zea mays; Ms: Musa sapientum; P: Pinus; Ch: Chamaerops humilis; W: Washingtonia; Ad: Arundo donax; Ao: Asparagus officinalis; Pc: Phoenix canariensis; Pd: Phoenix dactylifera; Pc: Phoenix reclinata ${ }^{\mathrm{c}}$ MAT alleles were obtained by Jurado et al. (2010). N.T: not tested

\section{Phylogenetic analyses}

Partial sequences of the IGS region from the isolates of $F$. proliferatum and closely related species listed in Table 1 were used for the phylogenetic analyses. Calculations of nucleotidic divergence and phylogenetic analysis were performed using PAUP $4.0 \mathrm{~b} 10$. An IGS sequence from $F$. verticillioides was used as the outgroup. Gaps were treated as missing data. The bootstrap method used the heuristic search with 1,000 replicates.

\section{Development of IGS type-specific PCR assays}

Two type-specific PCR assays were developed for each one of the two IGS types from $F$. proliferatum and $F$. globosum in the phylogenetic tree, type I and type II sequences, corresponding to the main clusters (Fig. 1). Primers were developed on the basis of the IGS genomic sequences alignment performed by the Clustal method using Dnastar (Lasergene, Madison, USA). The type I PCR assay with primers I-F (5' GGATAGCTCTAGGGTAGTT 3') and I-R (5' CTAGACRGACACRCAGGAR 3'), amplified a fragment of $250 \mathrm{bp}$. The type II PCR assay with primers II-F (5' CTGGTCGGGATGAGGG 3') and II-R (5' CTGGACG GACACRCAG 3') amplified a fragment of $300 \mathrm{bp}$. The aim of these PCR assays was to test if both types of sequences coexist in an isolate or not.

\section{Results and discussion}

In this work we used a partial sequence of the IGS region to study the phylogenetic relationships of a diverse sample of $F$. proliferatum strains and other closely related species.
The sequence analyzed contained 108 phylogenetically informative nucleotide positions and corresponded to the most variable part within IGS region in species of the $G$. fujikuroi complex (Mirete et al. 2004) and, therefore, was adequate for phylogenetic analysis at intraspecific level and among closely related species. The tree inferred on the basis of this sequence resolved two clusters corresponding to two IGS types supported by bootstrap values of $67 \%$ and $69 \%$, respectively (Fig. 1), termed IGS type I and type II. $F$. proliferatum and $F$. globosum strains grouped in both clusters, whereas $F$. concentricum and $F$. fractiflexum strains were included in the IGS type I cluster. This cluster also contained most of the $F$. proliferatum isolates (32 out of 44) and exhibited higher values of nucleotide divergence (maximum of $8.3 \%$ and $2.9 \%$ for type II and type I, respectively). No correlation of either type I or type II with geographic origin or host, which could be involved in the maintenance of both IGS clusters, was apparent. The tree topology contrasted with other previous reports (O'Donnell et al. 1998) and with the one reported in Jurado et al. (2010) with $E F-1 \alpha$ sequences, which contain only 42 phylogenetically informative nucleotides. In this case, isolates carrying type I or type II IGS sequences did not cluster in distinct groups. Moreover, isolates of $F$. proliferatum and $F$. globosum were resolved as different species, as expected, and supported by bootstrap values of $100 \%$ (Jurado et al. 2010).

Fig. 1 Tree inferred from the partial IGS region sequence data in $F$. proliferatum set with assigned bootstrap values. FC: $F$. concentricum; $\mathrm{FV}: F$ verticillioides; FFR: $F$. fractiflexum; FF: $F$. fujikuroi; $\mathrm{FP}: F$. proliferatum; FG: F. globosum. C.I: consistency index. Nt: nucleotides. Phylogenetically informative characters are indicated between brackets 


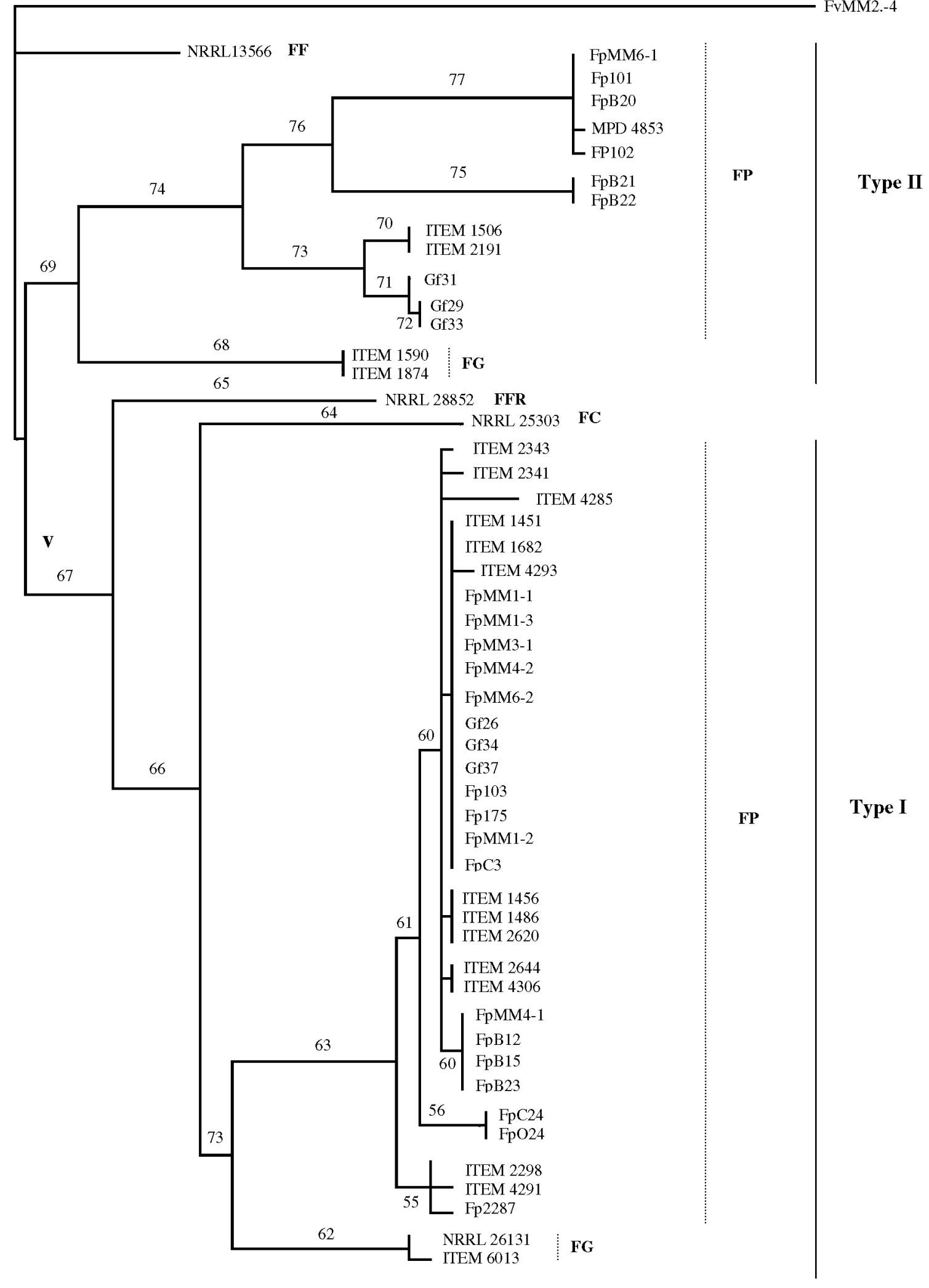


The existence of two subpopulations in the process of speciation could explain the divergence found in the IGS region. However, the tree inferred from $E F-l \alpha$ sequences did not resolve the same two groups of $F$. proliferatum strains. For example, isolates Gf29 and Gf33 (carrying type II IGS sequences) and Fp2287 (carrying type I IGS sequence), grouped together in the tree based on $E F-1 \alpha$ sequences (Jurado et al. 2010). In addition, this hypothesis cannot explain why the closely related species $F$. globosum clustered in a distinct group as separate species in both IGS types. Therefore, the absence of concordance between both sets of data indicated that the divergence found in the IGS rDNA sequence does not reflect speciation, and that both types of sequences might be non-orthologous IGS types. As we mentioned above, a similar situation was described for the ITS2 region in species of the G. fujikuroi species complex. In this case, the two non-orthologous type I and type II ITS2 sequences (a major and a minor), coexist in each individual (O'Donnell et al. 1998). Their maintenance might be facilitated if they were actually two different rDNA loci. According to Eickbush and Eickbush (2007), if ribosomal DNA units resulting from duplication events are located in different chromosomes, homogenization might be difficult since interchromosomal exchanges seem to occur less frequently than sister chromatid interchanges which could be considered as the basis of concerted evolution of units on individual chromosomes. In our work, we have analysed if the two IGS types detected in $F$. proliferatum and F. globosum occurred in the same individual or reflected intraspecific polymorphism. In order to test these hypotheses, two type-specific PCR assays were developed (type I and type II PCR assays) following a similar approach to the one previously used to reveal the presence of coexisting types of ITS1 sequences in individuals of the species Trichaptum abietinum (So Koo and Sung Jung 2002). The results obtained in the PCR assays performed are indicated in Table 1. Amplification bands were exclusively obtained for one of the two type-specific PCR assays (Fig. 2), indicating that only one IGS type was probably present in each individual. These results suggested that the two IGS types might have arisen by duplication in a common ancestor prior to the divergence of $F$. proliferatum and $F$. globosum, and followed by eventual fixation of one locus by birth-and-death processes. These processes seem also to be facilitated by dispersed location of genes (Rooney and Ward 2005). Alternatively, occasional hybridization between both species might have occurred, since evidences of incomplete reproductive barriers in this closely related group of species have been reported (Xu et al. 1995).

On the other hand, the maintenance of two divergent types in these two distinct species at the intraspecific level might be the result of inefficient concerted evolution (homogenization). Since the IGS region is not transcribed and translated, and thus, selective pressure is probably low, the process of homogenization of the IGS would basically rely on sexual recombination at the populational level. In addition, although MAT-1 and MAT-2 alleles, previously obtained in Jurado et al. (2010), are represented in both IGS types; thus, reproduction should be possible, a certain rate of clonal reproduction as well as the wide range of hosts colonized by $F$. proliferatum might limit sexual reproduction, thereby retarding the process of homogenization. Clonal reproduction exhibited by $F$. oxysporum has also been proposed to explain the maintenance of polymorphism within the IGS region found in this species at the intraspecific level (Appel and Gordon 1996; Campbell et al. 1997).

Analysis of the IGS region in other species of the $G$. fujikuroi species complex, such as $F$. verticillioides, did not reveal the presence of non-orthologous IGS types (data not shown). Nevertheless, more extensive studies should be carried out, sampling other more distantly related species of Fusarium.

The existence of non-orthologous types might lead to inaccurate phylogenies, as previously noted (O'Donnell et

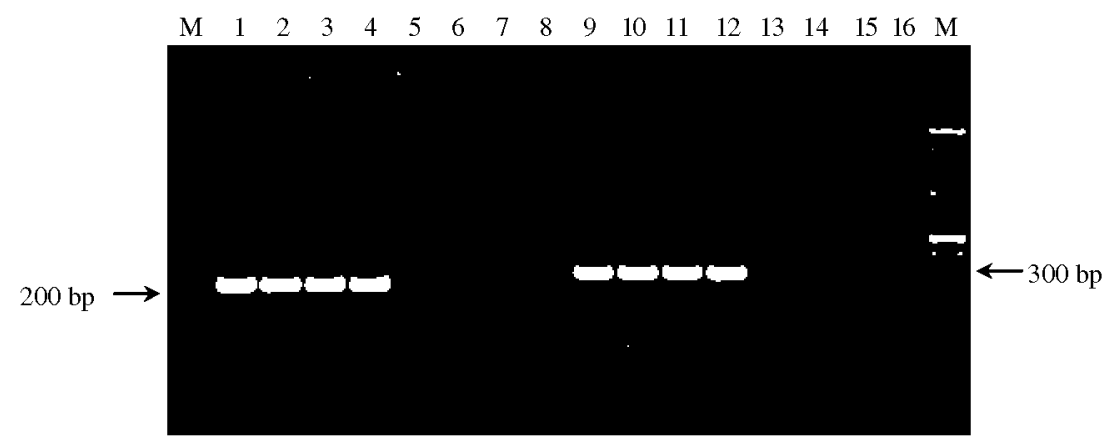

Fig. 2 Type-specific PCR assays carried out with primers Type I-F/ Type I-R and DNA from isolates of $F$. proliferatum $\mathrm{FpB} 12, \mathrm{FpC} 24$, ITEM 1682 and ITEM 2298 (lanes 1-4) and DNA from isolates of $F$. proliferatum Fp101, MPD 4853, FpB22 and Gf33 (lanes 5-8). Typespecific PCR assays carried out with primers Type II-F/Type II-R and
DNA from isolates of $F$. proliferatum $\mathrm{FpB} 12, \mathrm{FpC} 24$, ITEM 1682 and ITEM 2298 (lanes 9-12) and DNA from isolates of $F$. proliferatum Fp101, MPD 4853, FB22 and Gf33 (lanes 13-16). Lane M=100 bp DNA ladder plus (Fermentas, Lithuania) 
al. 1998; O'Donnell et al. 2000) and, additionally, it could negatively affect diagnostic assays based on these sequences. Since the IGS region has been broadly used for these purposes, the existence of non-orthologous IGS types in $F$. proliferatum and $F$. globosum, which might also occur in other Fusarium species, highlights that interpretation of the results concerning the use of IGS region should be undertaken with caution.

Acknowledgements This work was supported by the Spanish MEC (AGL-2010-22182-C04-01) and the UCM-CAM (GR58/08). P.M was also supported by FPI fellowships of the Spanish MEC.

\section{References}

Alves-Santos FM, Martínez-Bermejo D, Rodríguez-Molina MC, Díez JJ (2007) Cultural characteristics, pathogenicity and genetic diversity of Fusarium oxysporum isolates from tobacco fields in Spain. Physiol Mol Plant Pathol 71:26-32

Appel DJ, Gordon TR (1996) Relationships among pathogenic and nonpathogenic isolates of Fusarium oxysporum based on the partial sequence of the intergenic spacer region of the ribosomal DNA. Mol Plant Microb Interact 9:125-138

Campbell CS, Wojciechowski MF, Baldwin BW, Alice LA, Donoghue MJ (1997) Persistent nuclear ribosomal DNA sequence polymorphism in the Amelanchier agamic complex (Rosaceae). Mol Biol Evol 14:81-90

Dover G (1982) Molecular drive: a cohesive mode of species evolution. Nature 299:111-117

Eickbush TH, Eickbush DG (2007) Finely orchestrated movements: evolution of the ribosomal RNA genes. Genetics 175:477-485

Geiser DM, Jiménez-Gasco MM, Kang S, Makalowska I, Veeraraghavan N, Ward TJ, Zhang N, Kuldau GA, O'Donnell K (2004) FUSARIUM-ID v. 1.0: a DNA sequence database for identifying Fusarium. Eur J Plant Pathol 110:473-479

Jurado M, Vázquez C, Marín S, Sanchis V, González-Jaén MT (2006) PCR-based strategy to detect contamination with mycotoxigenic Fusarium species in maize. Syst Appl Microbiol 29:681-689

Jurado M, Marín P, Callejas C, Moretti A, Vázquez C, González-Jaén MT (2010) Genetic variability and fumonisin production by Fusarium proliferatum. Food Microbiol 27:50-57

Kosiak EB, Holst-Jensen A, Rundberget T, González-Jaén MT, Torp M (2005) Morphological, chemical and molecular differentiation of Fusarium equiseti isolated from Norwegian cereals. Int J Food Microbiol 99:195-206

Kvas M, Marasas WFO, Wingfield BD, Wingfield MJ, Steenkamp MT (2009) Diversity and evolution of Fusarium species in the Gibberella fujikuroi complex. Fungal Divers 34:1-21

Mirete S, Vázquez C, Mulé G, Jurado M, González-Jaén MT (2004) Differentiation of Fusarium verticillioides from banana fruits by IGS and EF-1a sequence analyses. Eur J Plant Pathol 110:515523

Nirenberg HI, O'Donnell K (1998) New Fusarium species and combination within the Gibberella fujikuroi complex. Mycologia 90:434-458

O'Donnell K, Cigelnik E (1997) Two divergent intragenomic rDNA ITS2 types within a monophyletic lineage of the fungus Fusarium are nonorthologous. Mol Phylogenet Evol 7:103-116

O'Donnell K, Cigelnik E, Nirenberg HI (1998) Molecular systematics and phylogeography of the Gibberella fujikuroi species complex. Mycologia 90:465-493

O'Donnell K, Kistler HC, Tacke BK, Casper HH (2000) Gene genealogies reveal global phylogeographic structure and reproductive isolation among lineages of Fusarium graminearum, the fungus causing wheat scab. Proc Nat Acad Sci USA 97:7905-7910

Rooney AP, Ward TJ (2005) Evolution of a large ribosomal RNA multigene family in filamentous fungi. Birth and death of a concerted evolution paradigm. Proc Nat Acad Sci USA 102:5084-5089

Smith GP (1976) Evolution of repeated DNA sequences by unequal crossover. Science 141:528-534

So Koo H, Sung Jung H (2002) Three nonorthologous ITS1 types are present in a polypore fungus Trichaptum abietinum. Mol Phylogenet Evol 23:112-122

Tóth A, Mesterházy A, Hórvath Z, Bartók T, Varga M, Varga J (2005) Genetic variability of European isolates of Fusarium graminearum complex. Eur J Plant Pathol 113:35-45

Waalwijk C, Koning JRA, Baayen RP, Gams W (1996) Discordant groupings of Fusarium spp. from sections Elegans, Liseola and Dlaminia based on ribosomal ITS1 and ITS2 sequences. Mycologia 88:361-368

Xu JR, Yan KY, Dickman MB, Leslie JF (1995) Electrophoretic karyotypes distinguish the biological species of Gibberella fujikuroi (Fusarium Section Liseola). Mol Plant Microbe Interact 8:74-84

Yli-Mattila T, Mach RL, Alekhina IA, Bulat SA, Koskinen S, KullnigGradinger CM, Kubicek CP, Klemsdal SS (2004) Phylogenetic relationship of Fusarium langsethiae to Fusarium poae and Fusarium sporotrichioides as inferred by IGS, ITS, $\beta$-tubulin sequences and UP-PCR hybridization analysis. Int J Food Microbiol 95:267-285 